\title{
Modeling of Contact Bounce in a Transient Electromagnetic Compatibility Test for the Analysis and Optimization of Nonlinear Devices
}

\author{
N. Lambrecht ${ }^{1}$, H. Pues ${ }^{2}$, Member, IEEE, D. De Zutter ${ }^{1}$, Fellow, IEEE, D. Vande Ginste ${ }^{1}$, Senior Member, IEEE.
}

\begin{abstract}
In this letter, we propose a methodology to include contact bounce, unavoidably occurring in a pulse generator, in the modeling of a transient electromagnetic compatibility (EMC) test. An example of such an EMC test is the RI 130 test, well-known in the automotive sector and used as case study in this letter. First, a detailed study of contact bounce of an electromagnetic relay is performed, leading to a novel modeling approach. Next, a multiconductor transmission line (MTL) equivalent is developed for the electrically large RI 130 test bench and concatenated with models for the load box and the device under test (DUT). Then, to apply and validate the advocated model, the behavior of the nonlinear DUT is simulated and compared to measurements under the RI 130 test conditions, showing good agreement. Finally, it is also shown that the overall model can be used to efficiently optimize the design of the DUT, making it more robust
\end{abstract}

Index Terms - transient immunity, contact bounce, nonlinear, stochastic process, modeling, optimization

\section{INTRODUCTION}

$\mathbf{F}$ OR designers of electronic circuits and equipment, it is of the utmost importance that early in the design phase, the electromagnetic compatibility (EMC) behavior of their novel devices and systems can be efficiently predicted. This avoids expensive iterations in later stages of the design cycle, costly and time consuming measurements and troubleshooting and hence, it reduces time to market of the products, leading to more cost-effective development. To accomplish this, modeling of EMC tests has become indispensable. However, there are many different types of EMC tests and almost every EMC test has to be modeled in a different way. The EMC test discussed in this letter is the RI 130 test [1], which is a broadband transient tests. RI 130 is a typical EMC test where contact bounce of an electromagnetic relay plays a significant role during the disturbance of the device under test (DUT). We propose a new modeling technique for the contact bounce, which we consider as a stochastic process. Together with a multiconductor transmission line (MTL) model for the electrically large RI 130 test bench, this results in an overall circuit equivalent, able to deal with active nonlinear components and easily integrated with advanced optimization and troubleshooting techniques. The method is thoroughly validated by means of measurements and its appositeness for rapid EMC analysis of the DUT, here

1 IBCN/Electromagnetics Group, Department of Information Technology, Ghent University/iMinds, TechnologieparkZwijnaarde 15, B-9052 Ghent, Belgium

${ }^{2}$ Melexis Technologies N.V., Tessenderlo, Belgium being a low drop-out voltage regulator (VR), and its subsequent optimization is illustrated.

The remainder of this letter is organized as follows. In Section II, the modeling techniques for each part of the RI 130 test setup, including the relay that is affected by contact bounce, are explained. Section III deals with the validation of the developed circuit model, via comparison with measurements using the nonlinear active DUT. Finally, some concluding remarks are formulated in Section IV.

\section{Methodology}

In this section, we discuss the RI 130 standard test [1] and, as a case study, we develop a circuit model for it. Particular attention is devoted to the modeling of the generator, including its relay that is affected by contact bounce.

\section{A. Description of the RI 130 test}

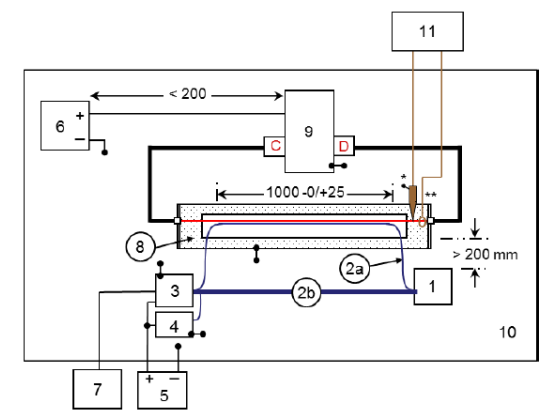

Fig. 1: The default RI 130 test setup [1], where 1=DUT, $2 \mathrm{a}=$ DUT circuit wire to be tested, $2 \mathrm{~b}=$ DUT wire harness, $3=$ load box, 4=artificial network, 5=power supply, 6=automotive battery, $7=$ DUT monitor, $8=$ coupling test fixture, $9=$ transient generator, $10=$ ground plane and $11=$ test point.

Although the methodology described in this letter is more generally applicable, as the RI 130 test is gaining importance, it is the focus of this letter and we start with its rigorous description. The default RI 130 test setup is depicted in Fig. 1. The test bench itself consist of a large ground plate and a coupling test fixture, which rests on this ground plane. An aggressor wire is mounted in the coupling test fixture and a single wire of the wire harness is placed on top of the aggressor wire in the coupling test fixture, while the other wires of the wire harness are placed at least $200 \mathrm{~mm}$ away from the coupling test fixture, so that no direct field coupling 


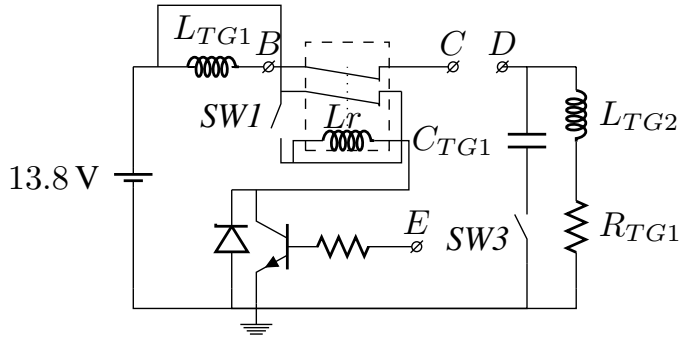

(a) Schematic of the transient generator.

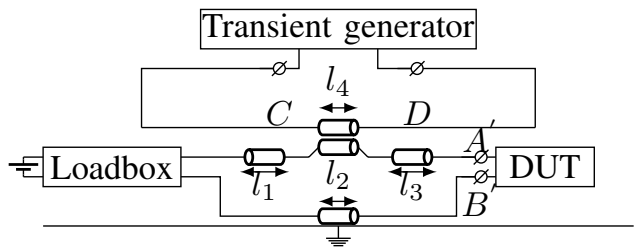

(b) The RI 130 test bench modeled with MTLs.

Fig. 2: The RI 130 test bench model.

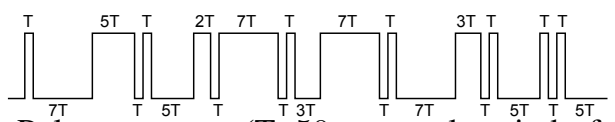



can occur between the aggressor wire and the remainder of the wire harness. As always, the wire harness connects the DUT with a load simulator (aka load box). In this particular EMC test, every wire of the wire harness is tested by placing it on top of the aggressor wire. The aggressor wire is excited by a transient generator, of which the schematic is shown in Fig. 2a. The transient generator comprises a passive charging circuit, which is triggered via an electromechanical relay. The required pulse sequence of $3.45 \mathrm{~s}$, depicted in Fig. 3, is applied to node $E$, saturating the transistor and, consequently, activating the relay. The components of the transient generator have the following values: $R_{T G 1}=39 \Omega, C_{T G 1}=100 \mathrm{nF}, L_{T G 1}=5 \mu \mathrm{H}$, $L_{T G 2}=100 \mathrm{mH}$. $L_{r}$ represents the relay's coil. The transient generator can be operated in several modes, depending on the settings of the switches SW1 and SW3. RI 130 encompasses four different modes, depending on whether SW1 and SW3 are closed or open. In [1], much attention is devoted to the case where SW1 is left open, this case is called mode 3 in [1]. Therefore, in this letter, we will apply our methodology to mode 3 .

\section{B. Modeling of the RI 130 test bench}

To avoid time-consuming full-wave simulations, we propose a multiconductor transmission line (MTL) approach to model the test bench. Thereto, we apply the same method as in [2]. Without loss of generality but for conciseness, we consider a wire harness consisting of only two wires. The corresponding equivalent cascaded MTL circuit is shown in Fig. 2b, where $l_{1}=0.3 \mathrm{~m}, l_{2}=1.2 \mathrm{~m}, l_{3}=0.15 \mathrm{~m}$ and $l_{4}=1 \mathrm{~m}$. The load box and the DUT are connected to the two ends of the wire harness. The load box is a passive impedance, readily characterized by means of S-parameter measurements or simulations, and it is described in the EMC test plan. At the other end of the wire harness, the DUT is connected. The two-terminal nonlinear DUT considered here, is described further in Section III.

\section{Modeling of the contact bounce}

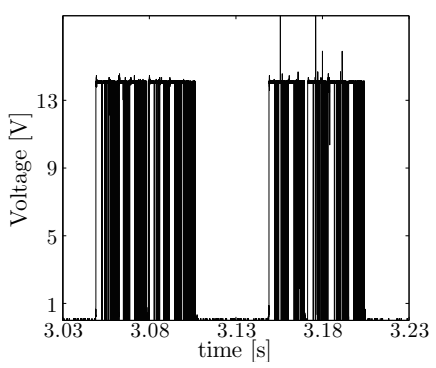

(a)

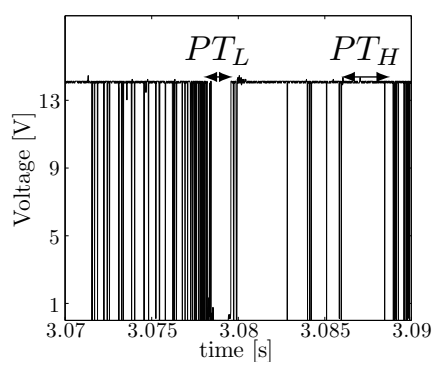

(b)
Fig. 4: (a) Typical voltage waveform between node $B$ and $C$ due to contact bounce of an electromagnetic relay in mode 3 when $L_{T G 1}=L_{T G 2}=C_{T G 1}=0$ and node $C$ and $D$ are short-circuited. (b) A zoom of the contact bounce Fig. 4a.

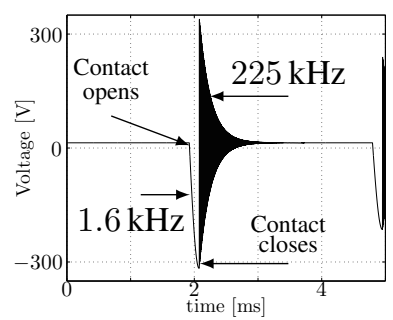

(a)

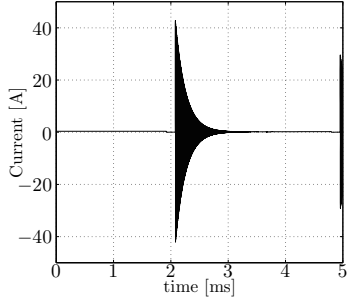

(b)
Fig. 5: Simulation of waveforms appearing in the transient generator of Fig. 2a, relevant to the contact bounce phenomenon: (a) voltage at node $D$ w.r.t. ground and (b) current through the aggressor wire for a worst case situation in mode 3, pulse A2-2.

It is known that contact bounce of a relay is unavoidable. When contact is made, two objects collide and there will be a rebound. The corresponding displacement waveform is not a sinusoid, but it has a damped behavior. If one is able to measure or simulate the displacement of the relay contact, then one can, e.g., make use of Prony analysis to directly estimate the frequency and magnitude of all exponentially damped modes [3]. However, in our case and often in EMC tests, the relay is standardized and we cannot change or even measure the displacement of the relay contact. Hence, we have chosen to characterize the contact bounce by measuring the voltage waveform directly across the relay contact (between nodes $B$ and $C$ in Fig. 2a). When the relay is activated in mode 3 by the transistor, the normal-closed (NC) relay contacts will open. So, the power supply will be disconnected from the relay's coil $\left(L_{r}\right)$, by which the NC relay contact rapidly closes again. Owing to the (unpredictable) contact bounce of the mechanical parts, this repeatedly opening and closing of the relay does not happen in a periodical fashion, but typically leads to a waveform as depicted in Fig. 4a, where the voltage waveform is shown for the last 
two pulses of the sequence shown in Fig. 3. When repeating the experiment many times, however, different waveforms with similar characteristics are obtained. Hence, these waveforms can be considered as a stochastic process. Recently in literature, polynomial chaos (PC) approaches [4], have been given a lot of attention to tackle variability in electronic design. Nonetheless, for the time-variant system described in this letter, PC becomes intractable (or even feasible). Therefore, we adopt a more pragmatic approach to deal with the stochastic process. In particular, we provide a good estimation of the worst case situation, which is of course of importance to the EMC engineer or designer. Fig. 4b zooms in on a smaller portion of the waveform. When doing so, it appears that two parameters "control" the shape of this waveform. $P T_{L}$ represents the time during which the relay contacts are closed during contact bounce and $P T_{H}$ represents the time when the relay contacts are open. We now consider these two parameters, $P T_{H}$ and $P T_{L}$, as random variables, defining the stochastic process. In case SW3 is closed, we are dealing with the so-called mode 3, pulse A2-2. In this mode, capacitor $C_{T G 1}$ is in parallel with the series coil $\left(L_{T G 2}\right)$ and resistance $\left(R_{T G 1}\right)$. We will further use mode 3, pulse A2-2 to apply our methodology. When all the relay contacts are at rest (e.g., during $P T_{L}$, see Fig. 4b) $L_{T G 1}, L_{T G 2}$ and $C_{T G 1}$ will be charged with a time constant $\tau=\left(L_{T G 1}+L_{T G 2}\right) / R_{T G 1}=2.56 \mathrm{~ms}$. On the other hand, when the relay contact is open, the LC tank $\left(L_{T G 2}, C_{T G 1}\right.$, $R_{T G 1}$ ) oscillates at $1.6 \mathrm{kHz}$. As can be seen from Fig. 5a, a maximum voltage will occur at a quarter of a period of the oscillating frequency $(=156.25 \mu \mathrm{s})$ after the relay contact opened. When the relay contact closes, $L_{T G 1}$ also affects the oscillation frequency, which then becomes $225 \mathrm{kHz}$. When the maximum voltage at node $D$ is reached, a maximum current will flow through the aggressor wire (see Fig. 5b) with peakto-peak values up to $80 \mathrm{~A}$. This in turn gives rise to a great deal of crosstalk from the aggressor wire to the victim wire that lays on top of it. In our proposed worst case scenario, the current through the aggressor wire and voltage at node $D$ should be as high as possible, as this leads to the worst crosstalk. Thereto, we choose $P T_{H}=156.25 \mu \mathrm{s}$, as we then close the relay contact when there is a maximum voltage at node $D$. The parameter $P T_{L}$ should be large enough such that the inductors can be fully charged. Typically, one could choose $P T_{L}=5 \tau=12.8 \mathrm{~ms}$. However, such a large time interval never really occurs during contact bounce. From measurements we derived that the maximum value for $P T_{L}$ during contact bounce is $2.72 \mathrm{~ms}$. So, we set $P T_{L}=2.72 \mathrm{~ms}$.

To implement this behavior using a circuit simulator, we replace the relay by a voltage controlled switch (see Fig. 6a). We apply the pulse sequence of Fig. 3 to this switch, where each individual pulse is now periodically switched on during a time interval $P T_{H}$ and switched off during a time interval $P T_{L}$. This leads to the worst case scenario. A detail of this signal, that is applied to the voltage controlled switch, i.e. again the two last pulses of the entire sequence, is shown in Fig. 6 b.

\section{MEASUREMENTS AND RESUlts}

To validate and illustrate the appositeness of our proposed model, we apply it to a nonlinear DUT and compare it with

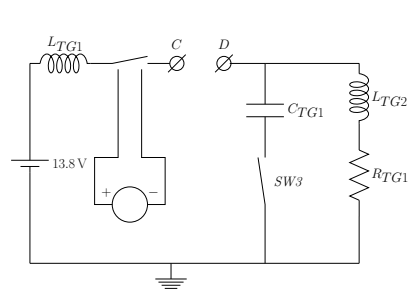

(a)

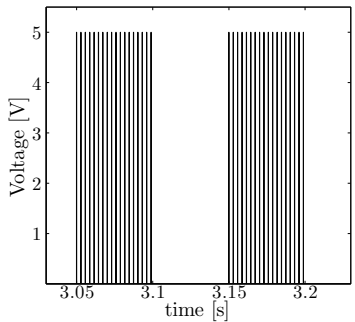

(b)
Fig. 6: (a) Proposed circuit model for the transient generator Fig. 2a. (b) Detail of the signal applied to the voltage controlled switch of Fig. 6a.

measurements. All measurement results are performed as described in [1], measuring the DUT during $60 \mathrm{~s}$, from which we select and show the worst situation, i.e., the particular $3.45 \mathrm{~s}$ period yielding the worst case result.

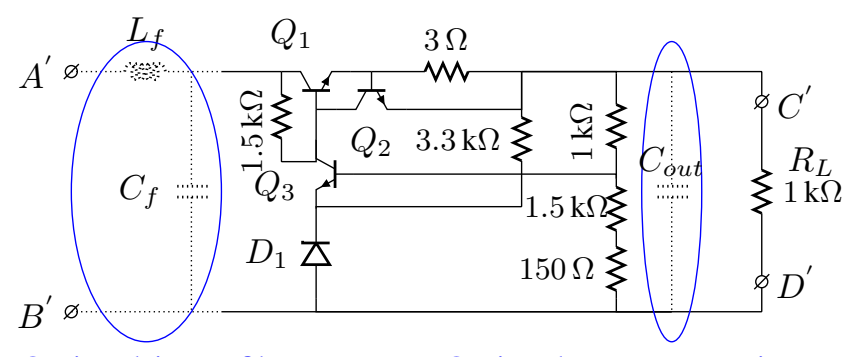

Optional input filter

Optional output capacitance

Fig. 7: Schematic of the controlled series voltage regulator. The optional components $\left(L_{f}, C_{f}, C_{\text {out }}\right)$ are omitted for the validation of Section III-A and their values are optimized in the application example of Section III-B.

\section{A. Validation of the model using a nonlinear DUT}

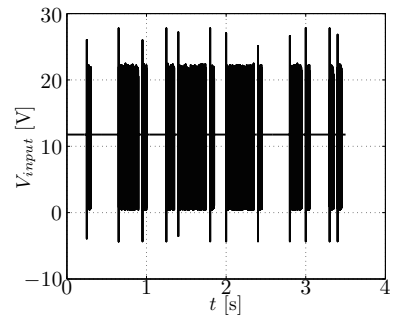

(a) Simulation.

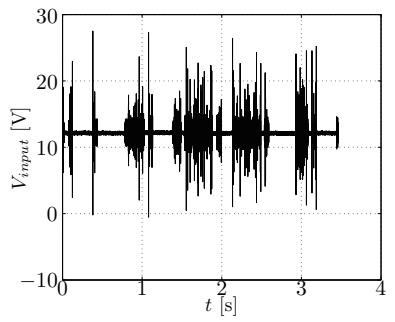

(b) Measurement.
Fig. 8: Input voltage of the voltage regulator shown in Fig. 7 during one full period of $3.45 \mathrm{~s}$ in mode 3, pulse A2-2.

The DUT is a low drop-out voltage regulator (VR) with schematic shown in Fig. 7. For clarity, the parasitics are not shown in the schematic, but they are include in the simulations, based on the datasheet of the components. In particular, the DUT is a controlled series regulator, converting an input voltage of $12 \mathrm{~V}$ to an output voltage of $5 \mathrm{~V}$. Bipolar junction transistors (BJT), i.e., the BC846 by NXP Semiconductor, are used in 


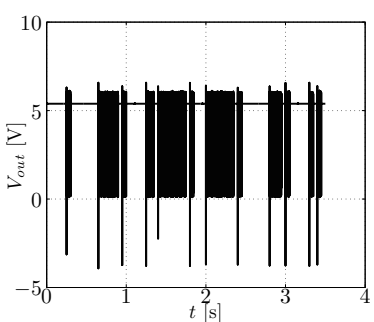

(a) Simulation.

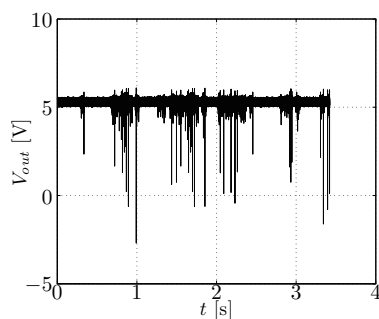

(b) Measurement.
Fig. 9: The output voltage of the voltage regulator shown in Fig. 7 during one full period of $3.45 \mathrm{~s}$ in mode 3, pulse A2-2.

this design. The zener diode is the BZX84C2V4 by NXP Semiconductors.

To validate our worst case model in concatenation with the proposed MTL model for the test bench, we measure the voltage at the input of the voltage regulator i.e., between nodes $A^{\prime}$ and $B^{\prime}$, during the test in mode 3, pulse A2-2 (Fig. 8b). Compared to the simulation with our novel model, it is clear that the (worst case) maximum amplitudes are quite accurately predicted without too much overestimation. When measuring the output of the voltage regulator i.e., between nodes $C^{\prime}$ and $D^{\prime}$, we get large negative peaks (Fig. 9). This is due to the zener diode. When the zener diode is forward biased, $Q_{3}$ will conduct current and $Q_{1}$ is in cut off, leading to a drop of the output voltage. During one full period of $3.45 \mathrm{~s}$, simulations with the advocated model (Fig. 9a) are compared with the measurements (Fig. 9b), again leading to the conclusion that the model correctly predicts the negative peaks. Due to the inherent unpredictability and complexity of the contact bounce, the tolerances on the components and their models provided by the vendors, one can of course not expect simulation and measurement results to coincide completely. In particular, tolerances on te zener diode cause a small discrepancy of the expected DC value.

For completeness, we mention that similar results are obtained in the so-called mode 3, pulse A2-1, i.e. when switch SW3 is open during the test.

\section{B. Optimization}

Whereas the model itself was validated in the previous section, it is also clear that this DUT would never pass the RI 130 test, as typically, one does not want the output voltage to deviate from $5 \mathrm{~V}$ by more than, say, $150 \mathrm{mV}$. To further illustrate the benefits of the proposed model, we will now use it to make the VR more robust. Thereto, an LC low-pass filter ( $L_{f}$ and $C_{F}$ ) at the input, and a capacitor $\left(C_{o u t}\right)$ at the output were added. Of course, for various reasons (parasitics, cost, mechanical, ...), we want the additional three components $L_{f}$, $C_{f}$ and $C_{\text {out }}$ to be as small as possible, whilst the output voltage remains with the interval $[4.85 \mathrm{~V}, 5.15 \mathrm{~V}]$. So, we are confronted with the following optimization problem:

$$
\begin{array}{ll}
\operatorname{minimize} & L_{f}>0, C_{f}>0, C_{\text {out }}>0 \\
\text { subject to } & 4.85 \mathrm{~V}<\left|V_{C}-V_{D}\right|<5.15 \mathrm{~V}
\end{array}
$$

This optimization problem is solved with the gradient optimizer embedded in the ADS circuit simulation of Keysight Technologies. Leveraging our novel equivalent circuit model, the complete optimization process only takes $8 \mathrm{~min} 42 \mathrm{~s}$ of CPU time and yields the following values: $L_{f}=40 \mu \mathrm{H}$, $C_{f}=20 \mathrm{nF}$ and $C_{\text {out }}=2 \mu \mathrm{F}$. To manufacture the optimized voltage regulator, the following commercially available values were selected: $L_{f}=47 \mu \mathrm{H}, C_{f}=22 \mathrm{nF}$ and $C_{\text {out }}=2.2 \mu \mathrm{F}$. As can be seen from Fig. 10, both in measurement and in simulation, the maximum derivation is reduced to less than $150 \mathrm{mV}$.

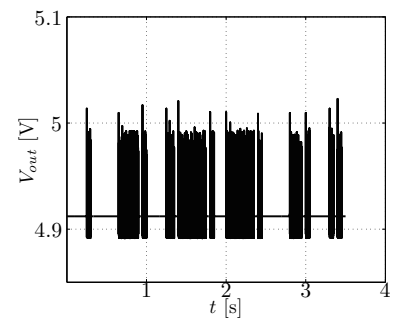

(a) Simulation

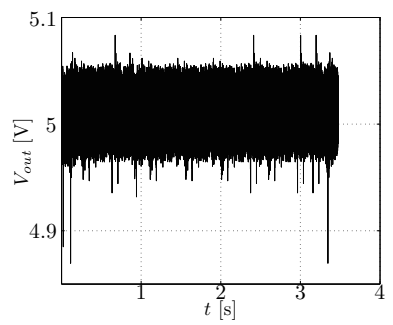

(b) Measurement
Fig. 10: The output voltage of the optimized voltage regulator during one full period of $3.45 \mathrm{~s}$ in mode 3, pulse A2-2.

\section{CONClusion}

In this letter, we have proposed a solution for the efficient circuit modeling of the stochastic behavior of contact bounce, observed during the RI 130 test. Additionally, the electrically large RI 130 test has been modeled with a MTL model. The combination of the MTL model and our novel proposed model of the contact bounce leads to a complete circuit model of the RI 130 test. With a nonlinear VR as DUT, this model was extensively validated by means of measurements and it was shown that it allows to efficiently and accurately predict the behavior of the DUT in the design phase. Moreover, we demonstrated that our proposed circuit model may be advantageously used for the optimization of DUTs, making them more immune against the RI 130 test conditions. It can be concluded that the advocated approach is useful to effectively troubleshoot and solve problems when contact bounce has to be taken into account in a transient EMC test.

\section{REFERENCES}

[1] FMC1278, Electromagnetic Compatibility Specification For Electrical/Electronic Components and Subsystems, www.fordemc.com/docs/download/FMC1278.pdf, Ford Std., 072015.

[2] N. Lambrecht, C. Gazda, H. Pues, D. De Zutter, and D. Vande Ginste, "Efficient circuit modeling technique for the analysis and optimization of ISO 10605 field coupled electrostatic discharge (ESD) robustness of nonlinear devices," IEEE Trans. Electromagn. Compat., vol. 58, no. 4, pp. 971-980, Aug 2016.

[3] J. Xiong, J. He, C. Zang, C. Liu, and L. Wang, "Influence of characteristic parameters on contact bounce in Reed systems of relays," 2008 Proceedings of the 54th IEEE Holm Conference on Electrical Contacts, Oct 2008.

[4] P. Manfredi, D. Vande Ginste, D. De Zutter, and F. G. Canavero, "Stochastic modeling of nonlinear circuits via spice-compatible spectral equivalents," IEEE Transactions on Circuits and Systems I: Regular Papers, vol. 61, no. 7, pp. 2057-2065, Jul 2014. 